\title{
MAKING THE INVISIBLE VISIBLE: MODELLING SOCIAL LEARNING IN A KNOWLEDGE MANAGEMENT CONTEXT
}

\author{
Henry Linger \\ School of Information Management and Systems \\ Monash University \\ Caulfield East, VIC 3145 \\ Phone: +61-3-9903 2260 \\ henry.linger@sims.monash.edu.au \\ Leoni Wame \\ Joint Systems Branch \\ Defense Science and Technology Organisation \\ Department of Defense \\ Canberra ACT 2600 \\ Phone: +61262658819 \\ Leoni.Warne@dsto.defence.gov.au \\ * Author for correspondence
}

\begin{abstract}
The articulation of Knowledge Management as an organisational strategy has occurred in the context of a radical shift towards an information based economy. The most significant aspect for organisations operating in the information economy is their ability to utilise the volumes of information that are now readily available without the constraint of media, geography or time. A critical factor for organisations is the speed at which they are able to productively process such information to enable the organisation to react rapidly to changes in their operating environments.

In this context organisation needs to produce and re-produce knowledge. The shift from information to knowledge is an acknowledgment of the significant role of the human actor in the process of transforming information into effective organisational outcomes. Social learning represents important processes that contribute to actors' ability to understand information, create knowledge from that information and share what they know. Social learning is therefore intrinsic to knowledge management.

In this paper we present a knowledge management architecture that supports a learning organisation. This architecture accommodates social learning and processes by which knowledge is internalised and externalised by individuals, work groups and the organisation as a whole. The architecture incorporates a model social learning based on the results of ethnographic studies and a model of leaming derived from knowledge management case studies. The architecture is not domain specific but can be applied to activity that can be characterised as knowledge work in an organisational context. As such the architecture can play a variety of roles; as a conceptual framework, as a diagnostic tool to identify breakdown and as a design tool for organisational change.
\end{abstract}

\section{INTRODUCTION}

The effective assimilation of knowledge into an organisation and the organisation's ability to learn and construct new knowledge is an imperative for many organisations in this 'knowledge millennium'. In addition organisations need to retain knowledge when individuals leave, or when situations change, to ensure the viability of the organisation in the longer term. This, in turn, has implications for the development of systems that manage organisational knowledge effectively. Many different research efforts have been launched to investigate and facilitate these processes (Brown \& Duguid, 1991; Davenport \& Prusak, 1998; Flood, 1999; Hildreth, Wright, \& Kimble, 1999; Ward, 1998).

Knowledge exists in the minds of individuals, groups and in organisations. It is, therefore, linked to the human mind, its cognition, reasoning and understanding, but it is shaped by the goals of the organisation or group of which the individual is a member. Knowledge management, therefore, is about the acquisition, construction, transfer, and sharing of the knowledge assets within an organisation, aimed at achieving the organisation's objectives. However, the term 'knowledge management' can mean different things to different people; from informal processes that mediate the transformation of an individual's implicit knowledge into explicit knowledge that can be shared; to the complex knowledge databases that facilitate formal organisational processes and capture, store and disseminate vital corporate knowledge.

Clearly there is a myriad of knowledge management interests that occur along the continuum between informal processes and technological artefacts. Our position in this continuum is that knowledge management needs to develop organisational knowledge assets (mainly people), support them with information technology and other tools, and enhances the processes by which individuals, groups, and the entire organisation learns. In our view, knowledge management needs to be concerned with cultural, organisational, procedural, and human factors as well as with tools and technologies. Together these form the basis of an organisation's integrated knowledge management strategy. 
The purpose of this paper is to present a series of models that can represent the whole continuum, emphasising the process of organisational social learning that we believe underlies successful knowledge management in organisations. We present a general model of learning, a specific architecture for social learning and a generalised knowledge management architecture for a learning organization that represents the continuum discussed above. Through these models the paper explores how social learning is incorporated into a knowledge management framework and how this extended framework contributes to the development of a learning organization.

The models presented in this paper are the result of collaboration between the authors who have worked respectively in knowledge management and social research that focussed on social learning. The collaboration is based on our belief that social learning is tightly coupled to knowledge management. These models draw on knowledge management models derived from a number of case studies (Linger et al., 2000; Linger et al., 1998) and the ethnographic studies conducted by the Enterprise Social Learning Architectures (ESLA) research team at the Defence Science and Technology Organisation (DSTO) (Warne, 2000). In this paper we focus on activity that can be characterised as knowledge work that is not domain specific.

The composition of this paper is the following. Section 2 presents a model of learning and discusses the role of social learning in this model. Section 3 introduces the ESLA task, models the finding as an architecture for social learning and maps the findings onto the learning model. The section also presents a summary of the ESLA findings. Section 4 presents a knowledge management architecture that supports a learning organization while the following section links the three models. Section 6 makes concluding remarks regarding the models.

\section{A MODEL FOR REPRESENTING SOCIAL LEARNING}

In this section, we introduce a model of learning that embodies social learning. This model is presented in Figure 1 below. The model is based on the assumption that knowledge work underlies learning and that such work is focussed on a task or a specific activity. This changes the perspective from an organisational view to a focus on work practices. In this paper we use the term "actor" to denote an individual who is performing a particular role, emphasising our focus on work practices. The notion of "actor" defines work as a social activity, performed through human agency, but maintains the primacy of work roles in executing a task. Implicit in our use of the term actor is a person who has the necessary skill and knowledge to perform the role. This leads to a definition of a community of practice that includes those actors who are engaged directly in performing some aspect of a task (Wenger, 1998). The task boundaries are defined by the community, but authorised by the organization, thus allowing members of the community to be distinguished from other potential stakeholders in the task. Organisationally however, actors engaged in a particular task can (and usually are) also members of other communities that exist either within a particular organisation or are independent of that organisation.

The model involves three perspectives and three sites of discourse. The micro level discourse is the personal and private that allows each individual, or actor, to maintain their own individual/private tacit and explicit knowledge and understanding of a task or activity. The actor can use this framework to perform the task as well as to explore the boundaries of his or her knowledge. We consider all work to be social and represent this by showing more than one actor involved in the task. This allows a multitude of interpretations of how the task is performed, ranging from each actor needing to perform different aspects to collectively complete a single instance of the task, through to the same task being performed many times by the same or different actors and instances of the task being performed at different times and/or places. Each actor then has the opportunity to publicise their understanding of the task by making their knowledge available to the community. The discourse at the meso level can be mediated with artefacts or through verbalisation that encodes aspects of each actor's private knowledge. The intention is to provoke discussion and debate, not to impose a particular view. It is the actor who determines when and what is made available to the community. This sharing with the community represents the personal and public perspective. The result of discussion within the community is a consensual perspective that is expressed through a minimal framework representing the current shared and negotiated understanding of the task that all actors would be required to apply to the performance of that task (Levitt and March, 1988). The discourse at the macro level represents the organisation's formal and explicit definition of the task. 


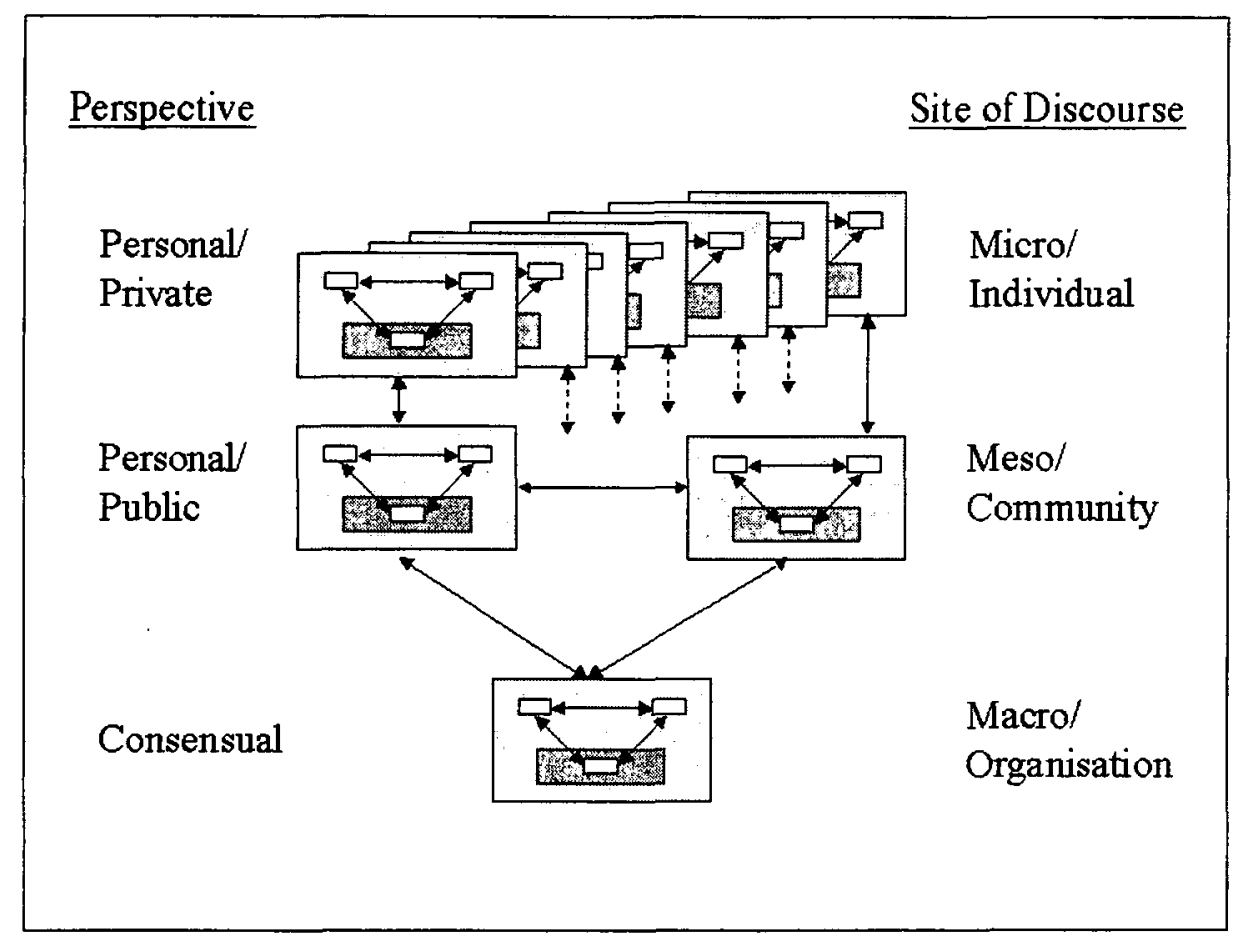

Figure 1. A model of learning (adapted from Linger, 2000)

Social learning is represented by the arrows in Figure 1. The arrow between the micro and meso levels indicates an actor's engagement in a social process that represents that actor's participation in a community of practice. The arrow that links the actors within the community represents the social learning that includes the varieties of social and cultural interactions through which actors share and acquire knowledge. In this context, social learning also includes formal aspects of learning, such as training and records management, as the focus is on processes rather than the activity itself. The arrows that link the meso level to the macro level define the mechanisms by which an organisation formalises social learning into sanctioned procedures to achieve organisational outcomes. These mechanisms also form part of the organisation's social learning as they are manifested in the organisation's decision making structure built around more formal social processes such as committees and governing bodies.

A significant feature of this model is that social learning is represented by double-headed arrows. This indicates that there is feedback in. every learning process. This means that the actor makes a contribution to the social or cultural process and gains from their engagement with the process. In the context of social learning, the value for the actor is their evolving knowledge of the activity of the community of practice. The nature of the social learning mechanisms contributes significantly to the culture of the organisation and the prevailing organisational values. As a specific example, the feedback in social learning, indicated by the double-headed arrows in the model, define a learning organisation. Without this feedback, or if it is ineffectual, the organisation reverts to a hierarchical bureaucracy.

The boxes in Figure 1. indicate a body of knowledge $(\mathrm{BoK})$ that is pertinent to the perspective adopted and the level of discourse (Iivari and Linger, 2000). As shown in the diagram this knowledge has an internal structure represented by a task-based knowledge management framework (Linger, 2000). This indicates that some aspects of the body of knowledge at each level of the model can be expressed as explicit artefacts. Such representation allows the design of computer-based support systems to facilitate the learning processes as well as task performance. However, it needs to be stressed that the learning model is generic and is not dependent on this internal framework of the body of knowledge.

Applying the results of the ESLA studies to this model of learning, it is apparent that the learning processes and strategies that were identified in the studies correlate to the interaction between and within the levels of the model. This is discussed in detail in section 3 below. 


\section{THE ENTERPRISE SOCIAL LEARNING ARCHITECTURES (ESLA) TASK}

The ESLA task is a four-year research study investigating social learning within the Australian Defence Organisation. Social learning, in this context, refers to learning done in or by a group, an organisation, or any cultural cluster and includes:

- the procedures by which knowledge and practice are transmitted across posting cycles, across different work situations and across time; and

- the procedures that facilitate generative learning - learning that enhances the enterprise's ability to adjust to dynamic and unexpected situations and to react creatively to them.

The immediate aim of this research is to understand the issues inherent in building leaming, adaptive and sustainable organisations. The long-term objective, however, is to develop architectures that will support the development of information systems that guide and enhance organisational learning. In this way, social learning is inextricably interwoven with knowledge management.

The ESLA study began in June 1998 and has acquired data from several different ADO settings. The first setting was a 'tactical' single service headquarters, where a pilot study was conducted to determine the feasibility of the project's aim and methods. The research team returned to this Headquarters in April and May 2001 to validate that the findings remained stable over time. The second setting was a joint (all three services and civilians) 'strategic' headquarters within the main Australian Defence Headquarters. Currently, further studies are underway in a single-service strategic headquarters. More information on the ESLA study findings is presented in a separate article in this issue (Warne et al., 2001). The methodologies used and research conducted has been reported in depth elsewhere (see Agostino, Gori, \& Warne, 1999; Ali et al, 2000; Warne, 1999; Warne, 2000)

\section{An emerging architecture for social learning}

The progressive findings of the ESLA studies, reported in other papers (see papers listed above), represent the collective research results of all the different settings studied to date. The focus is on the findings that are likely to be relevant to all organisations, not just military ones. These findings are multi-layered and allowed the research team to pinpoint a number of environmental and cultural issues, processes and strategies that facilitate effective social learning and knowledge management. In this paper we have conceptualized these results into the architecture represented in Figure 2 below.

The ESLA findings can be collectively conceptualised as a learning toolset of actions, processes and strategies that the organisation engages to facilitate its activities to achieve organisational outcomes. This toolset impacts social learning in four distinct ways; as motivators, enablers, challengers and inhibitors. Our position is that each tool has a greater or lesser impact on each of these effectors of social learning. The organisational values arise from the balance of these organisational effectors, but in turn, the values inform these effectors and how they are balanced. These reciprocal and interdependent relationships emerge from the findings of the ESLA research. While motivators form a sound foundation and enablers provide the bricks or building material, the values are the mortar that binds them all together. Challengers and inhibitors are environmental or personal factors that impede or erode the organisation's continued robustness.

The architecture also indicates that the organisation's performance influences its values. This introduces a temporal dimension into the dynamic as there is often a temporal distance between an outcome and when it is reflected back unto the organization by the external environment. This is indicated in the diagram by the arrows originating with the organisational outcomes.

An example of a study finding mapped to this architecture is inquiry and reflection. An important element of generative learning is for organisational members to be able to engage in dialogue that is open and is based on inquiry and reflection. A supportive communication climate is a prerequisite for such dialogue and it requires learning how to recognise defensive patterns of interaction that undermine learning(Ali et al., 2000; Senge, 1992). However, an additional and obvious requirement for such dialogue is having the time to engage in it. On numerous occasions, the ESLA team encountered comments from staff at both the strategic headquarters that there is little time to reflect, learn from experiences (irrespective of whether the experience was a successes or failure) and generally discuss work matters. Workload was attributed as a factor preventing people from setting some time aside for thinking and reflection and such comments were often made with an indication of bitterness. On the other hand many of the processes at the tactical headquarters, from mission debriefs to tactics meetings and training, demonstrated that they recognised the importance of inquiry and reflection at a group level. As well as improving the quality of the reflection, group 
reflection also contributes to the development of a common identity and a shared understanding amongst the participants.

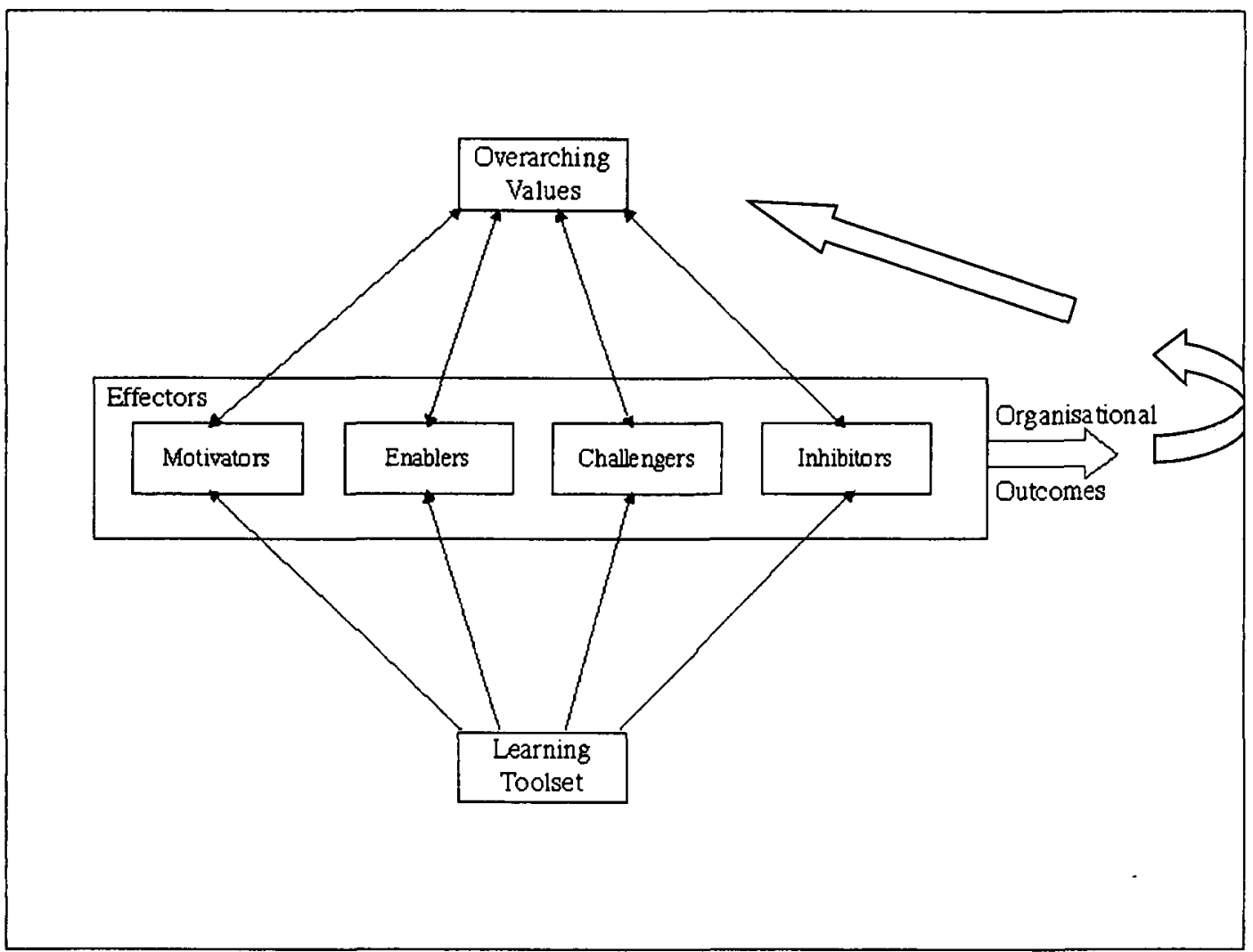

Figure 2. An architecture for social learning

Reflection as a tool is an enabler because it allows the actors to remove themselves from the actual performance of the activity. Reflection provides the actors with the space and distance they need to better understand the activity they are engaged in. Reflection as a motivator allows the actors to learn from their engagement in the activity, with the intention of instigating some form of improvement of the activity. This level of control is empowering. However, reflection requires time that takes the actor away from the actual performance of an activity. The challenge is how to accommodate reflection in the actors' work practices. Reflection is also an inhibitor, as it is often an unacknowledged and unrewarded activity. The organisational outcome is determined by how these four effectors are applied in a particular situation. It is the net balance that defines the outcome. Moreover the net balance also impacts on the overarching organisational values. For example, if, on balance, reflection is predominantly a motivator and enabler, it contributes to defining empowerment as an organisational value. Simultaneously, empowerment, as a value, exerts an influence on how the tool, reflection, is viewed in the organisation.

Some of the other processes and strategies identified in the ESLA studies that can be considered part of the toolset in the architecture include:

- timely and relevant handover and induction;

- building a common identity;

- strong goal alignment;

- effective workplace design;

- facilitating personal networks;

- systemic understanding;

- team building;

- public recognition and award systems; 
- IT infrastructure;

- Mutually beneficial performance management systems;

- facilitating accurate perceptions of the organisation;

- apprenticeships and legitimate peripheral participation;

- peer review and cross-peer learning;

- mentoring and/or buddying systems;

- the use of social networks and social organisation;

- the use of mediating artefacts and bridging agents;

- bricolagel;

- the judicious use of protocols at meetings and briefings; and

- effective formal information flows and records management.

\section{Applying ESLA finding to the learning model}

It is apparent that the learning processes and strategies that were identified in the studies correlate to the interaction between and within the levels of the learning model discussed in section 2 . It would appear that the social learning processes are dominant in the interaction between the individual and community level of the learning model, and at the community level itself.

Processes that enable new members of the community to gain access to the community's knowledge and its practices include:

- timely and relevant handover and induction;

- effective workplace design;

- systemic understanding;

- facilitating personal networks;

- team building;

- facilitating accurate perceptions of the organisation;

- bricolage;

- apprenticeships and legitimate peripheral participation; and

- mentoring and/or buddying systems.

The community also engages in processes that evolve its knowledge and practices so as to maintain the community's ability to perform its activities in changing circumstances. These processes include:

- encouragement of dialogue based on inquiry and reflection;

- building a common identity;

- $\quad$ strong goal alignment;

- effective workplace design;

- facilitating personal networks;

- systemic understanding;

- team building;

- public recognition and award systems;

- IT infrastructure;

- Mutually beneficial performance management systems;

- facilitating accurate perceptions of the organisation;

- peer review and cross-peer learning;

- the use of social networks and social organisation;

- bricolage;

- the judicious use of protocols at meetings and briefings; and

\footnotetext{
', Bricolage is the ability to "make do with "whatever is to hand" "(Brown and Duguid 1991:10). Even when working on formal tasks, people will use whatever resources, social and material, that are available to them. When 'standard procedures' no longer seem adequate, they will improvise. People will develop models and knowledge that will enable them to achieve their goals (Brown and Duguid 1991; Agostino et al 1999). This also implies that the actors have been given enough autonomy to be able to improvise and adapt their practice, as the circumstances require.
} 
- effective formal information flows and records management.

While these processes have a community focus, they also serve an important cultural and learning function for the individual, especially a new community member. For the individual, these processes reinforce acceptable forms of behaviour, encourage knowledge sharing and reuse, reflective practice as well as constructing an identity based on membership of the community.

A viable organisation requires effective interaction between its diverse communities of practice. The processes that support this are:

- systemic understanding;

- the use of social networks and social organisation;

- the use of mediating artefacts and bridging agents;

- the judicious use of protocols at meetings and briefings; and

- effective formal information flows and records management.

It is apparent that the social learning processes and strategies can, and are, used in different ways depending on which level in the learning model they are applied to. The ESLA studies highlight that such distinctions are somewhat abstract as in practice a learning process is simply used by the actor to address a variety of needs. The ESLA studies also clearly identify and distinguish new members of the community and the learning strategies adopted by the community to induct these novices as full members. It is instructive to note that the same processes are used by the community on an ongoing basis, albeit for a different purposes, and are not restricted to induction of new members. For example mentoring is an important strategy for inducting new members into the community. But equally it is a process that requires established members to articulate aspects of the community's knowledge. This process of "making the invisible visible" is in itself a learning process for established member and is indicative of the critical role of social learning in a learning and adaptive organisation that shares its knowledge.

The ESLA studies also highlight that there are fewer learning processes that facilitate the interaction between the community level and the organisational level of the model. This deficit is problematic in that it calls into question the process by which the consensual view is developed. This has considerable impact operationally as it potentially establishes a conflict between the community's understanding of the task, based on experiential knowledge, and the organisational view derived from an abstract understanding of the task. From the perspective of social learning, the ESLA studies indicate that the organisation uses mediating artefacts and bridging agents, meeting protocols and formal information flows and records management as strategies to support interaction between the organisation and its communities. These strategies fit into formal structures and cannot work alone to support learning. The interaction between the community and organisational levels was not explicitly studied in the ESLA project to date, so that the observations above are an interpretation and extrapolation of the existing studies.

\section{A summary of ESLA findings}

The findings suggest that social learning will flourish where the influences of power, trust, forgiveness and sharing are positively applied to the construction of knowledge. These factors provide a fertile ground for learning by allowing people to speak honestly, share information and offer suggestions without fear of ridicule or retribution ((Hoffmann \& Withers, 1995)

The positive outcomes that arise from the effectors in the learning architecture nurture and are nurtured by the overarching organisational values that include:

- empowerment (where empowerment of staff also makes them accountable);

- trust (which entails mutual respect);

- forgiveness (in terms of allowing personnel to take reasonable risks, forgiving mistakes and facilitating knowledge construction on the basis of lessons learnt)

- cultural cohesiveness (in terms of common identity, shared goals and a shared understanding)

- commitment (which includes a mutual commitment and loyalty from the employee to the organisation as well as the organisation to the employee)

- openness of the decision making process and

- a culture of information sharing.

What has emerged from the ESLA project is the paramount need for the ADO to facilitate knowledge transfer and the construction of objectified organisational knowledge. To this end the ADO has adopted diverse social learning 
processes that aim to engender a learning organisation. The studies that have been conducted indicate how central social learning is to the organisation's effective performance while also highlighting the difficulty in applying these processes to the diverse activities that are undertaken. The challenge for the ADO is to adopt these values in the process of transforming the $\mathrm{ADO}$ into a learning organisation. These values need to underpin the enculturation of individuals and communities within the $\mathrm{ADO}$ as well as to be expressed in the formal structures and processes of the organisation. The diffusion of values from the macro level of the learning model to the meso and micro levels provides a consistent narrative that underlies social learning and knowledge management in the organisation

\section{A KNOWLEDGE MANAGEMENT ARCHITECTURE FOR A LEARNING ORGANISATION}

A knowledge management architecture that supports a learning organisation, shown in Figure 3 below, accommodates actors in their ability to act effectively as individuals, and members of a community of practice, within an organisation. It also incorporates processes that enable the actors to perform their tasks, learn, and engage effectively in knowledge work as well as the means to objectify and document their understanding of their tasks.

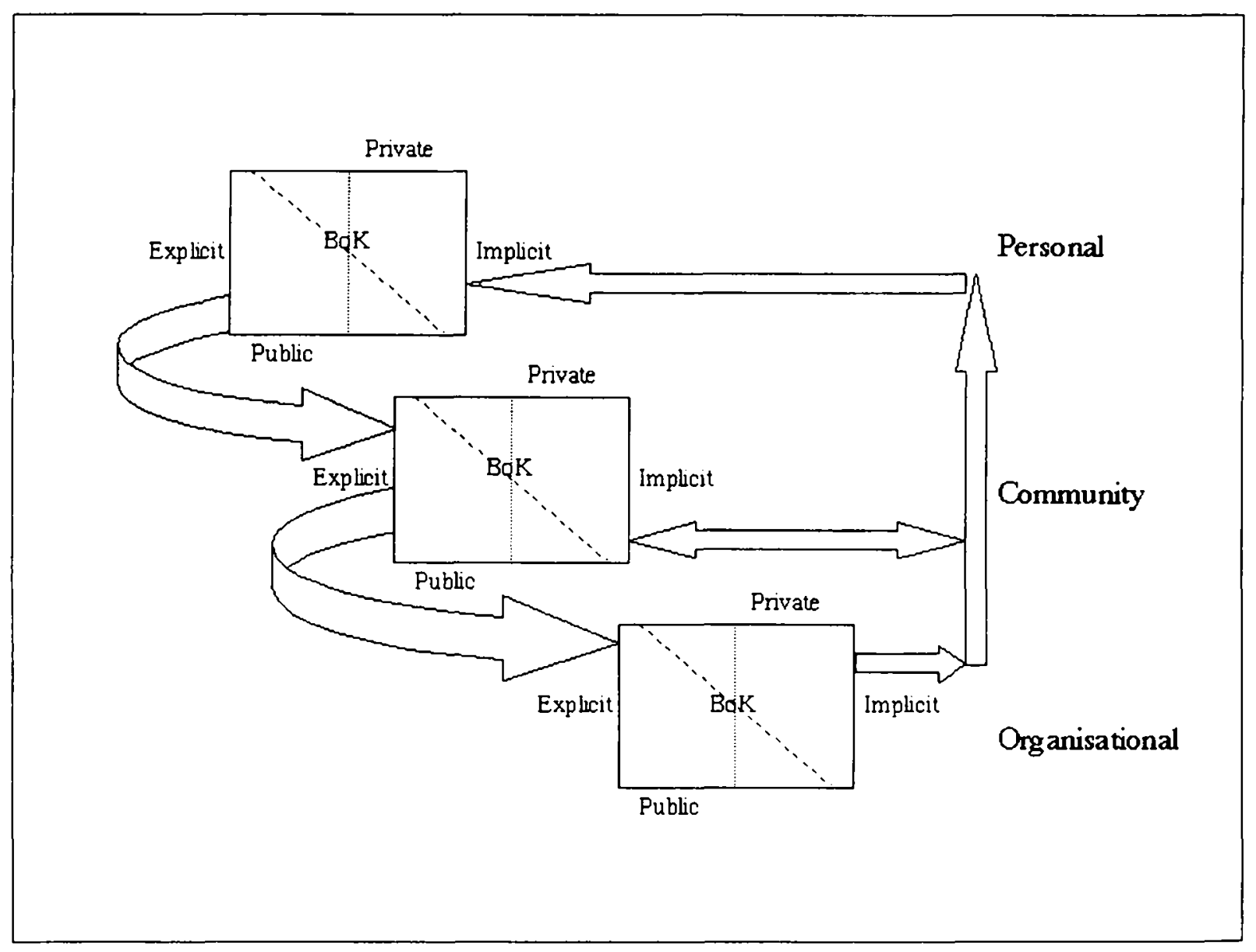

Figure 3. A knowledge management architecture for a learning organisation

The significance of this architecture is that it represents two largely antithetical processes through which the organisation maintains its viability. The left side indicates the social, and cultural processes through which actors engage in knowledge work, including knowledge constnuction, reconstruction and sharing. To a large extent this incorporates the formal and informal social learning processes and strategies. The architecture shows the left loop as predominantly a process flowing from the individual to the community to the organisation. The processes however are bidirectional providing feedback between all levels. This loop is essential because it is the means by which individuals and communities are able to act effectively, by adapting the formal, authorised definition of the task to changing cir- 
cumstances. Moreover this loop is informed by the over-arching organisational values and facilitated by the toolset identified above.

On the other side is the formal managerial command structure that establishes the authorised procedures through which activities are performed. On the whole, there is little if any input from individual actors into formulation of these procedures although the architecture indicates that communities could have a formal role. The importance of this loop is that it establishes the context in which activities take place and contributes to the implicit knowledge that actors bring to the activity in terms of their understanding of the organisational imperatives that underlie the task. Without this understanding diverse communities that constitute the organisation would not be able to work toward identifiable organisational goals. This also represents the full knowledge management continuum referred to in the Introduction to this paper. It is our contention that the social and cultural aspects of knowledge management predominate through the left-hand side of the model, and the formalised organisational systems and tools dominate the right-hand side of the model.

The architecture borrows the 3 levels of the learning model to identify the sites of discourse that provide the granularity necessary for a rich, multi-layered narrative of an organisation. The level of discourse is represented by the body of knowledge (BoK) that is relevant to that level. Included in the representation are two of the dimensions of the BoK that influence learning within this architecture. The private/public dimension is resonant with the learning model (Figure 1.) in that it identifies what knowledge is available for sharing. At the individual level it refers to what knowledge is available to all actors. At the community level it defines what the community communicates to the organisation about its activities. At the organisational level, this dimension deal with what information the organisation releases to the public at large. This is consistent with the learning model in that internal processes must exist that decide what knowledge and information is private or public. The implicit/explicit dimension relates to making sense of the world, and changes in it, and expressing and objectifying that understanding. It represents processes necessary to internalise learning. In the context of this architecture, both the left and right loops interact with the BoK as a whole rather than a particular point within these dimensions. Nevertheless, it is relevant that social learning does in fact accommodate knowledge that is useful and can be shared (mainly public and explicit). On the other hand, the objective and formal information that is the command loop needs to be assimilated and internalised in order to make sense of these organisational imperatives (mainly private and implicit). The discussion of these processes is outside the scope of this paper.

The ability of the loops to work synergistically appears to be dependent on the implementation of all components of both loops. As discussed above, social learning processes are not extensively employed between the community and the organisation. This weakens the left loop and results in an over-reliance on the right loop in determining how tasks are performed. This reinforces the hierarchical, bureaucratic organisational structure to the detriment of other possibilities implied by an adaptive learning organisation

For example one of the ESLA findings was that the building of a common identity through cultural cohesion was one of the tools for social learning. At the personal level, this related to how closely actors identified with their workplace and working colleagues. It was clear from the differences in the single service and joint settings that there were much stronger bonds in the single service environments than in the joint environment. At the community level, this cultural cohesion impacted on the shared understanding of the work activity and a shared vision for the organisation. At the organisational level this transformed into mission statements and stated organisational values. These same statements and values form part of the public image of the organisation. This public image then feeds into the community level of the organisation motivating the community and influencing how they perceive their goals and objectives. For example, some of the negative public perceptions of the Defence organisation that were aired in the media during the ESLA studies had an immediate impact on the morale and perceived self-value of communities within the ADO. They perceived themselves to be powerless and under-valued, and this directly impacted on how they viewed the goals and the objectives of their community.

Although in organisations where the right loop is not transparent, the public image can be interpreted at the community and individual level as rhetoric that is at odds with experiential perception. In organisations where the rhetoric matches experience the right loop between the community and individual acts to improve morale and job satisfaction by improving the individual's understanding of their role in the organisation. Not surprising, this is in itself empowering and can be seen as propagating the organisation's values.

The importance of this interpretation of the knowledge management architecture is that it reveals the potential for contradiction that are inherent in organisations as well as the complexity that arises from multiple viewpoints. What it also identifies implicitly is the processes that are necessary to integrate these viewpoints and a synthesis for the contradictions between the viewpoints. 


\section{LINKS BETWEEN THE THREE MODELS}

The Knowledge Management Architecture shown in Figure 3 adopts an organisational perspective that is sufficiently granular to reveal the organisation's internal functioning. Each level of this architecture implicitly incorporates both the Learning Model (Figure 1) and the Architecture for Social Learning (Figure 2), albeit with different interpretations at each level. At the individual level, the model of learning represents the actors' engagement with the organisation through their participation in communities of practice. The social learning architecture, when applied to the individual level, represents the actors' interpretation of the organisational values and identifies what subset of the learning toolset applies to them and how these tools impacts their ability to contribute to organisational outcomes. At the community level, the learning model identifies the members of the community and their contribution to the community as well as how the community influences the viability of the organisation. The social learning architecture allows the community to adapt the formal organisational values to their activities and to adopt a toolset necessary to meet their organisational requirements. At this level, the community needs to overtly balance the effectors to produce the defined outcomes. At the organisational level, the learning model identifies the communities and its members and their contributions. In terms of the social learning architecture, as discussed above, values are often formally and explicitly stated and the toolset focuses on the formal training and learning programs conducted by the organisation. The organisation also explicitly identifies the effectors, and their net effect, in terms of the stated aims, objective and such measures as key performance indicators.

The explicit/implicit dimension of the BoK in the knowledge management architecture relates to the information and knowledge that exists at each level and relates to the ability of the leaming organisation to manage knowledge in support of both its ability to perform work and to learn. The interpretation of this dimension, in the context of the architecture, can be limited to encoded and informal information and knowledge as the focus is on the social and cultural processes, which contribute (positively or negatively) to learning. Thus the emphasis is on knowledge that can be articulated in some way. The meaning of the public/private and explicit/implicit dimensions of the BoK varies at each level of the architecture. For example at the individual level, implicit could refer to the experiential knowledge an actor acquires through work performance. By contrast, implicit at the organisational level could refer to the cultural significance of myth expressed through the re-telling of stories relating to episodes in the organisation's history (Shanks, 1990)

\section{CONCLUSION}

In this paper, we have presented a series of models that can represent the whole continuum of interests and issues related to the social and cultural aspects of knowledge management. This continuum ranges from the transformation of individuals' implicit knowledge to the implementation of formal processes and tools that store, support and provide corporate knowledge within organizations. The organisational social learning that underlies successful knowledge management in organizations was mapped unto these models. The ESLA studies are valuable in that they empirically reveal the social processes and cultural norms that make the organization viable. Importantly the data highlights how significant social learning is to the organization, while ironically it shows that these processes remain largely unacknowledged in their contribution to organisational viability. The studies "make the invisible visible".

'Architecture', like knowledge management, has a range of meanings and uses. It can simply mean a high level description (model) of the system to be built. To others, it is conceptual or logical as opposed to physical. To others still, 'architecture' is 'requirements' whereas to some, it is simply a set of 'principles' (Zachman, 1999). To the authors of this paper, it is used as a tool to facilitate understanding of complex issues. It is apparent that the models and architectures presented in this paper play multiple roles in our research. They have been, or could be, used as:

- a conceptual framework that informs the conduct of a study in any setting;

- an analytic tool to organise and interpret data

- a framework to summarise findings

- a diagnostic tool to identify breakdown

- a design tool for organisational change

The longer term aim of our research is to utilise the models presented in this paper to define architectures for support systems that facilitate knowledge sharing, and particularly the ability to make more explicit the knowledge and practice of the community. Such systems would be an integral aspect of the transformation of the organization into a learning organization. 


\section{REFERENCES}

Agostino, K., Gori, R., and Warne, L. (1999) The Enterprise Social Learning Architectures Task: Context and Conclusions from the Pilot Study (Restricted DSTO-RR-0160), Canberra: DSTO.

Ali, I., Pascoe, C., Warne, L., Gori, R., and Agostino, K. (2000) Enabling Learning: A Progress Report on the Enterprise Social Learning Architecture Task, Proceedings of the 5th International Command and Control Research and Technology Symposium, Canberra, Australia

Brown, J. S., and Duguid, P. (1991) Organisational learning and communities-of-practice: Toward a unified view of working, learning and innovation, Science, 2, 1, 40-57.

Davenport, T. H., and Prusak, L. (1998) Working Knowledge: how organisations manage what they know, Harvard Business School Press, Boston, Massachusetts.

Flood, R. L. (1999) Rethinking the Fifth Discipline: Learning within the unknowable, Routledge, London.

Hildreth, P., Wright, P., and Kimble, C. (1999) Knowledge Management: Are We Really Missing Something?, Information Systems - The Next Generation: Proceedings of the 4th UKAIS Conference, York, UK, 347-356.

Hoffmann, F., and Withers, B. (1995) Shared Values: Nutrients for Learning, in Learning Organisations: Developing Cultures for Tomorrow's Workplace, Productivity Press, Portland, Oregon.

Levitt, B. and March, J.G. (1988) Organisational Learning; Annual Review of Sociology, 14: 319-340.

Iivari, J. and Linger, H., 2000, "The Characteristics of Knowledge Work: A Theoretical Perspective", Proceedings of the Americas Conference on Information Systems, AMCIS'2000, Long Beach California, August.

Linger, H., Burstein, F., Kelly, J., Ryan, C. and Gigliotti, P., 2000, "Creating a Learning Community Through Knowledge Management: The Mandala Project", Proceedings of IFIP WP8.3 Conference on DSS and Knowledge Management, Stockholm, July.

Linger, H., 2000, "Re-inventing the Firm: Organisational Implications of Knowledge Management", 4th International Conference on Business Information Systems, BIS'2000, Poznan, Poland.

Linger, H. Burstein, F. Zaslavsky, A. Aitkin, C. and Crofts, N; 1998, "An innovative organisational tool for epidemiological research"; European Journal of Epidemiology, 14:587-593

Senge, P. M. (1992) The Fifth Discipline: the Art and Practice of the Learning Organization, Random House, Sydney.

Shanks, R. (1990) Tell me a story: a new look at real and artificial memory, Macmillian Publishing Co. New York, USA.

Ward, A. (1998) Knowledge Management, http:/www.zdnet.co.uk/itweek/brief/1998/27/knowledge/ http:/www.zdnet.co.uk/itweek/brief/1998/27/knowledge/.

Warne, L. (1999) A Sociotechnical Approach to Social Learning Analysis in the Australian Defence Force, in E. Coakes, R. Lloyd-Jones, and D. Willis (eds.), The New Sociotech: Graffiti on the Long Wall, Springer-Verlag for the British Computer Society, London.

Warne, L. (2000) Understanding Organisational Learning In Military Headquarters: Findings From A Pilot Study, Australian Journal of Information Systems, 7, 2, 78-87.

Warne, L., Ali, I. M., Pascoe, C., and Agostino, K. (2001) "A Holistic Approach to Knowledge Management and Social Learning: Lessons Learnt from Military headquarters" Australian Journal of Information Systems, Special Edition on Knowledge Management.

Wenger, E. (1998), Communities of Practice: Learning, Meaning and Identity, Cambridge University Pres, Cambridge, UK

Zachman, J. A. (1999) A framework for information system architecture, IBM System Journal, 38, 2/3, 454-470. 